\title{
El modelo de la alternancia y la desigualdad educativa territorial en la educación en Colombia
}

The alternation model and territorial educational inequality

in education in Colombia

\section{Doris Yaneth Herrera Monsalve ${ }^{1}$ Universidad Santo Tomás}

Recibido: 14.12 .2020

Aceptado: 28.12.2020

"La universal superior de los inferiores se unirá a la universal inferioridad de los superiores para construir un mundo donde ninguna inteligencia podrá reconocerse en su semejante" (Ranciére, 2018, pág.76)

\section{Resumen}

Este artículo pretende evidenciar la desigualdad educativa territorial que existe en Colombia, en especial, en el marco de la coyuntura de la emergencia sanitaria nacional por causa del coronavirus COVID-19, declarada mediante resolución 385 del 12 de marzo de 2020 por el Ministerio de Salud y Protección Social y de conformidad a lo manifestado en la circular 20 del Ministerio de Educación Nacional, por tanto, se pretende problematizar el "modelo de alternancia" propuesto por el gobierno nacional. Este artículo pretende abrir opciones para repensar a los sujetos que van a la escuela -estudiantes y docentes- como lo plantea Southwell (2014): "los múltiples modos en que los sujetos enseñantes asumen, viven y piensan su tarea, y los problemas, desafíos y utópicas que se plantean en torno de ella” (p. 166).

\footnotetext{
1 dorisherreram@gmail.com https://orcid.org/0000-0001-6740-8770
} 
Por tanto, el modelo de la alternancia presenta la posibilidad de una relación multidimensional, y se espera que este texto sea un disparador para conversaciones entre los hacedores de la política pública y los sujetos que asisten a las instituciones educativas como una oportunidad para debatir y comprender "el orden social" (Ranciére, 2018).

En suma, la ruta de este articulo plantea un interrogante para abrir diálogos en el sistema educativo del centro y la periferia ¿Cuáles son esos artefactos que posibilitaran traspase de la sinrazón no igualitaria?, en términos de Ranciére (2018): “el amor a la dominación obliga a los hombres a protegerse unos de otros, en el seno de un orden convencional que no puede ser razonable porque no está hecho más que de la sinrazón de cada uno” (p. 134).

El texto se organizó en dos grandes apartados. El primero de ellos, busca hacer una presentación somera que describe y ubica geográficamente a dos municipios en ColombiaBogotá y Arauca-, al parecer muy disimiles por la extensión y la ubicación geográfica que aplican los mismos indicadores que da cuenta de los discursos hegemónicos que han estado presente en el continente latinoamericano y que han socavado la libertad en términos de Ranciére (2018): “es más cómo compararse, establecer el intercambio social como ese truque de gloria y desprecio en donde cada uno recibe superioridad en contrapartida de la inferioridad que confiesa. Así la igualdad entre seres razonables vacila en la desigualdad social" (p. 134). En un segundo apartado, se plantea que la alternancia plantea una medida desde una mirada univoca perdiendo de perspectiva la riqueza que da el entramado social del sistema de educación en Colombia y pone sobre la mesa una oportunidad para abrir mundos y caminos distintos para cumplir con la permanencia escolar y establece un desafío para familias, docentes, niños y jóvenes en la ampliación de estrategias en clave de la concepción de la justicia como un asunto ético de la sociedad colombiana que no pierda de vista la educabilidad capaz de producir desarrollos según las características singulares de los sujetos (Martinis, 2016).

Palabras claves: Desigualdad educativa, Arauca, Bogotá, COVID-19, Alternancia. 


\section{Abstract}

This article aims to show the territorial educational inequality that exists in Colombia, especially in the context of the national health emergency due to the COVID-19 coronavirus, declared by resolution 385 of March 12, 2020 by the Ministry of Health and Social Protection and in accordance with what is stated in circular 20 of the Ministry of National Education, therefore, it is intended to problematize the "alternation model" proposed by the national government. This article aims to open options to rethink the subjects who go to school students and teachers- as stated by Southwell (2014): "the multiple ways in which teaching subjects assume, live and think about their task, and the problems, challenges and utopians that arise around it" (p. 166). Therefore, the alternation model presents the possibility of a multidimensional relationship, and it is expected that this text will be a trigger for conversations between public policy makers and subjects who attend educational institutions as an opportunity to debate and understand "The social order" (Ranciere, 2018). In short, the route of this article raises a question to open dialogues in the educational system of the center and the periphery. What are those artifacts that will make it possible to pass on the nonegalitarian unreason ?, in terms of Ranciere (2018): domination forces men to protect themselves from each other, within a conventional order that cannot be reasonable because it is made only of the unreason of each one" (p. 134).

The text was organized into two large sections. The first of them seeks to make a brief presentation that describes and geographically locates two municipalities in ColombiaBogotá and Arauca-, apparently very dissimilar due to their extension and geographical location, which apply the same indicators that account for the hegemonic discourses that have been present in the Latin American continent and have undermined freedom in terms of Ranciere (2018): “it is more like comparing oneself, establishing social exchange as that trick of glory and contempt where each one receives superiority in counterpart of the inferiority that confesses. Thus, equality between reasonable beings wavers in social inequality" (p. 134). In a second section, it is proposed that alternation poses a measure from a univocal point of view, losing perspective on the richness that the social fabric of the education system in 
Colombia gives and puts on the table an opportunity to open different worlds and paths to comply with the school permanence and establishes a challenge for families, teachers, children and young people in the expansion of strategies in the key of the conception of justice as an ethical issue of Colombian society that does not lose sight of the educability capable of producing developments according to the unique characteristics of the subjects (Martinis, 2016).

Keywords: Educational inequality, Arauca, Bogotá, COVID-19 and alternation.

\section{La desigualdad territorial}

La desigualdad educativa territorial que existe en Colombia, en especial, en el marco de la coyuntura de la emergencia sanitaria nacional por causa del coronavirus COVID-19, declarada mediante resolución 385 del 12 de marzo de 2020 por el Ministerio de Salud y Protección Social y de conformidad a lo manifestado en la circular 20 del Ministerio de Educación Nacional, por tanto, se pretende problematizar el modelo de alternancia propuesto por el gobierno nacional desde una mirada de la exclusión social y las desigualdades educativas. Entendiendo, que con el aislamiento obligatorio se pone de manifiesto que los sectores sociales más vulnerables serán los mayores afectados para recibir los servicios como la educación y que pone sobre la mesa la discusión de lo poco solidaria que son las sociedades, (Dubet, 2015) plantea que la crisis de las solidaridades, entendidas como el apego a los lazos sociales que nos llevan a desear la igualdad de todos, ha intensificado las desigualdades (...); en una sociedad plural donde las culturas y los individuos esperan que se los reconozca como autónomos y singulares, se vuelve imprescindible construir los espacios y las escenas que permitan decir lo que tenemos en común, a fin de aceptar nuestras diferencias.

Con la evidencia empírica y con los casos presentados por algunos medios de comunicación, se observa que existe una fractura en el tejido social y en especial de los servicios básicos para las poblaciones con mayor dificultades para la comunicación, se mantiene o aparece en el colectivo colombiano una nueva escisión social en términos de dentro/fuera, entre quienes

Volumen 1. Número 2. Julio - Diciembre 2021 
tiene internet y un computador para trabajo en casa y entre quienes no poseen estos nuevos artefactos que permiten una comunicación constante con sus docentes, por tanto, pone a niños y jóvenes en mayor vulnerabilidad frente al acceso a quienes no logran contar con dichas herramientas, pero el hacedor de política pública olvida los modos de vida de los diferentes territorios, y se pone en evidencia los fallos de las políticas públicas inclusivas.

Por tanto, las opciones que se establezcan para los territorios deben articular nuevos diseños y estrategias para encontrar caminos que garanticen el derecho a la educación para todas las comunidades en términos de (Southwell, 2016) "articulaciones de elementos, traducciones de otros discursos y de nuevas relaciones y tramas que se dan en el marco de mecanismos denominados de hibridación” (p. 174) y que los lineamientos de la política pública se hacen desde una mirada lineal y pierden de vista las particularidades regionales y locales y sobre todo la voz de los docentes, quienes han sido en la coyuntura los que han brindado y utilizado diversas herramientas para mantener a los estudiantes atentos a su proceso de enseñanza.

Y tanto los medios de comunicación como el gobierno nacional a través del Ministerio de educación; se han encargado de decir que los docentes no están formados para el uso de las tecnologías, es suma, pareciera que todo el sistema es homogéneo y las recetas se aplican sin ninguna discusión, como lo plantea Ranciére "Se representan a sí mismo como sociedades homogéneas en las cuales el ritmo vivo y común de la multiplicación de mercancías e intercambio allanó las viejas divisiones sociales de clases y hace participar a todo el mundo en los mismos goces y libertades" (p. 15). Muy lamentable esa posición que se evidencia en los discursos y deja de lado, la voz del docente como elemento central en la garantía del desarrollo a la educación y en la posibilidad que tiene el oficio de enseñar radica en la dimensión vincular, afectiva y relacional del trabajo con personas que realizan los docentes, la noción de posición docente implica también una dimensión ético-política en la que se combinan apuestas por lo que el trabajo de enseñar y las nuevas generaciones pueden hacer, iniciativas de magnitud y otras relativamente pequeñas; miserias, complejidades y dificultades del oficio; todas las cuales componen un territorio tensionado y difícil de asir desde una única mirada (Soutwell, 2016, p. 167). 
Lo anterior, no sólo ocurre en los lineamientos para el preescolar, sino para los cuatro niveles en los que está organizado el sistema escolar colombiano, que, a su vez, tienen diversidad de lecturas y dentro de cada nivel existen desafíos propios en cada territorio. Se puede decir, que el sistema aún es insuficiente para lograr la cobertura universal en términos de indicadores de coberturas, tanto en el nivel transición y en el nivel educación básica secundaria. En las grandes ciudades como Bogotá, Medellín, Cali y Bucaramanga, el sistema está conformando por un alto número de colegios privados con un alto porcentaje de absorción de estudiantes y contribuye a generar circuitos diferenciadores en una sociedad de niveles de estratificación socioeconómica muy marcado en las prácticas cotidianas, a continuación, una breve descripción de los niveles:

. Educación Preescolar: Corresponde a la educación impartida a los niños y niñas menores de 6 años. Abarca tres años, aunque solo uno es obligatorio para las entidades públicas (transición).

. Educación básica: Comprende los ciclos de básica primaria que incluye los grados de primero a quinto y básica secundaria que va del sexto al noveno grado.

. Educación media: comprende los grados diez y once.

. Educación superior: corresponde a la educación técnica, tecnológica y universitaria, con una duración que de 1 a 7 años.

En el desarrollo normativo después de la Constitución Política de 1991, en aras del reconocimiento a poblaciones en condición de vulnerabilidad, los artículos 67, 68 y 69 de la Constitución Política de Colombia denominan la educación como un derecho de la persona y un servicio público que tiene una función social. Los responsables de asegurar la educación son el Estado, la sociedad y la familia. En los artículos queda manifiesto que la educación será obligatoria entre los 5 y los 16 años de edad y será gratuita en las instituciones educativas oficiales y, además, que será el Estado quien deberá ejercer vigilancia, propender por garantizar la cobertura, la calidad, la permanencia y el acceso al sistema escolar. 
Posteriormente, la Ley 115 de 1994 (Ley General de Educación), define y desarrolla la organización y la prestación de la educación formal en sus niveles de preescolar, básica (primaria y secundaria) y media, no formal e informal.

Para efectos de este escrito se hará un análisis general de la educación básica primera y segundaria, sin mucha mención a la educación superior, en tanto, ahondar en dicho nivel implica mayor tiempo de análisis y detalle, a partir de evidenciar con datos estadísticos los diferentes niveles de desigualdad educativa y territorial que existe entre las dos regiones de estudio. Los lineamientos decretados por la pandemia en especial, frente al tema de garantizar el derecho se educación se pone de manifiesto la desigualdad socio-económica un factor que afecta el acceso y se evidencia en la desigualdad educativa, poniendo sobre las discusiones temas estructurales e históricos en el país; que existe y es invisibilizado por los hacedores e implementadores de la política social; en tanto, en muchas ocasiones sólo se mencionan de manera despectiva para menospreciar a las personas en condición de vulnerabilidad y con una idea muy asociada a que es por pereza que no logra la inserción de un sistema desigual.

Por ello, para adentrarnos a la discusión es necesario mencionar que Colombia tiene un índice de Gini de 0,57\% es el segundo país más desigual de la región y el octavo a nivel mundial según datos de la CEPAL y el Banco Mundial, por tanto, comprender el fenómeno de barreras de acceso en la educación supone varias tensiones para la política pública y se consolida como una barrera más fuerte para los niños y jóvenes de regiones apartadas y que hacían parte de los territorios nacionales, en tanto, el tema de la distribución del ingreso se mantiene en un 0,57 y según cifras de la Dirección Nacional de Planeación -DNP- existen serias diferencias y barreras de acceso entre las cabeceras municipales y las zonas rurales que evidencian brechas visibles entre las Ciudades y los Centros poblados. 


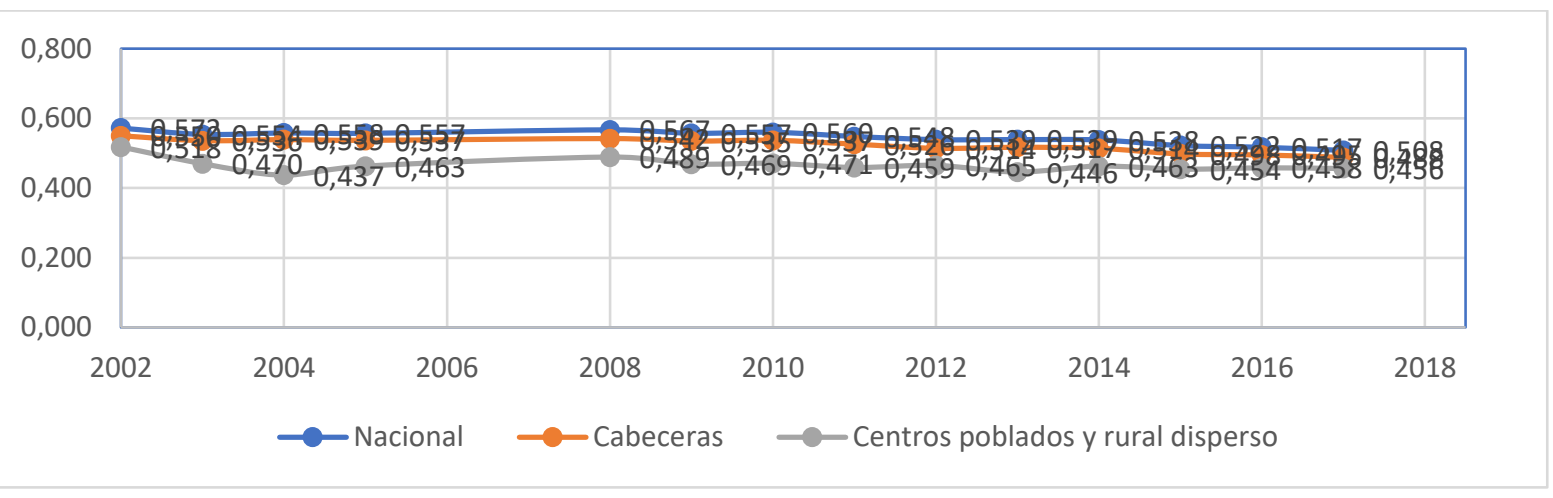

Gráfico 1. Índice de Gini en Colombia

Fuente: Elaboración propia a partir de datos de la Gran Encuesta de hogares, Dane, 2017

Por lo anterior, se hace necesario hacer una comprensión de las desigualdades territoriales y entender la educación desde una perspectiva histórica como un medio que equipara las oportunidades de las personas en los contextos sociales y un vehículo para la equidad en contextos de exclusión y discriminación social se empieza a identificar una profunda diferenciación entre unos y otros (porque no decir entre clases sociales); es decir, aspirar a una sociedad en que todas las personas tengan igualdad de oportunidades para vivir una vida digna (Chiroeu. 2012, 2013) dejando claro que existen causas de tipo histórico y cultural en la educación en Latinoamérica requiere una revisión de tipo estructural (Puigróss, 1996) y posibilitando la discusión del carácter social, sumado que estas concepciones son producto de las construcciones de los relatos asociados a experiencias.

Por tanto, es necesario aclarar el concepto de inclusión en una identidad determinada supone la exclusión de otros, la definición de una frontera o límite más allá de la cual comienza la otredad (Dussel, 2000), por tanto, es necesario cuestionar el relato de la inclusión, es decir, la narrativa que sostiene que la expansión del sistema escolar moderno es la única manera, y la mejor, de ilustrar al pueblo y democratizar las sociedades (Ibidem, 2000), esa idea en términos de Ranciére (2018) “atribuyen a la escuela el poder fantasmático de realizar la igualdad social o, por lo menos, de reducir la fractura social y en sociedades donde eran capaz de confesar la división de clases" (p. 15) y en nuestras sociedades no tienen la capacidad de reconocer esas divisiones y en cambio se representan como sociedad homogéneas y todo el mundo participa en los mismos goces y libertades y por ende, establecen lineamientos homogéneos para todos. 
Por ello, el tema de revisar y derrumbar barreras para el acceso y en consecuencia con la necesidad de abrir otras opciones y sobre todo en el marco de la pandemia y la posibilidad de vislumbrar las diferentes brechas existentes a lo largo y ancho de Colombia y no sólo en el sector educativo, sino en diferentes ámbitos y lograr dar un rol protagónico a la comunidad educativa.

A partir de las diferencias entre centros poblados y las capitales se presentará una descripción de Bogotá y Arauca para comprender las dinámicas educativas y las diferencias territoriales y así lograr una visión general de la situación social y educativa que a traviesan los niños y jóvenes en el marco del aislamiento obligatorio determinado por el gobierno a partir del 24 de marzo de 2020, y poner en la agenda pública a la escuela como esa institución capaz de resolver la desigualdad existente.

Por lo anterior, es importante caracterizar dos territorios disimiles en su composición, pero con ideas muy comunes sobre la aplicación de los lineamientos establecidos por el ente rector en el marco de la Pandemia; las ciudades de Bogotá y Arauca respectivamente, también, a manera ilustrativa dar elementos al lector sobre la ubicación geográfica con particularidades del sector educativo principalmente. La ciudad de Bogotá:

está situada en la sabana del mismo nombre, sobre el altiplano cundiboyacense de la cordillera Oriental de los Andes) a una altitud de $2650 \mathrm{msnm}$ y en sus puntos más altos hasta 4050 msnm. EL Distrito Capital de Bogotá tiene un área total de 1732 km² (incluyendo la localidad de Sumapaz) y un área urbana de 307,39 km² (en 1996). (Molina, 1996, p. 302)

Es la capital del país y cuenta con excelentes índices de acceso a los servicios públicos, así como la ciudad que concentra el mayor número de instituciones de educación con mayor nivel de calidad de la educación en el país. 
En cuanto al sector educativo, se encontró que la matrícula del sector oficial es de 785.171 estudiantes y se concentra en la zona urbana con un 96,7\% equivalente a 759,302 niños y con un $3,3 \%$ en la zona rural equivalente a 25,869 estudiantes según los datos del Sistema Integrado de Matrículas -SIMAT- con corte al 28 de febrero de 2019. Bogotá como distrito capital cuentan con una tasa de cobertura escolar ${ }^{2}$ del 87,3\%, según datos de la Secretaría de Educación Distrital -SED-, a continuación, encontrará la tasa de cobertura por el nivel educativo.

\begin{tabular}{|l|l|l|l|l|}
\hline GLOBAL & PREESCOLAR & BÁSICA PRIMARIA & $\begin{array}{l}\text { BASICA } \\
\text { SECUNDARIA }\end{array}$ & $\begin{array}{l}\text { MEDIA } \\
\text { VOCACIONAL }\end{array}$ \\
\hline 87,3 & 72,4 & 88,4 & 92,4 & 82,3 \\
\hline
\end{tabular}

Tabla 1. Tasa de cobertura escolar en Bogotá Fuente: Secretaría de Educación del Distrito Elaborado por DICE - SDP

El segundo, es un municipio ubicado en el oriente en Colombia, se denominado Arauca, capital del departamento de Arauca, "está localizado sobre el margen sur del río Arauca. Limita con Venezuela al norte, con la cual está conectada mediante el Puente Internacional José Antonio Páez y se comunica por vía terrestre hacia el centro de Colombia mediante la Ruta de los Libertadores, que une a las ciudades de Caracas y Bogotá” (Wiki, 2020) y se encuentra a una distancia de 720 kilómetros de la ciudad de Bogotá. Frente a las cifras, según las proyecciones del censo del año 2005 del Departamento Administrativo -DANE-, en dicho municipio existen 94,377 habitantes de los cuales 82,341 habitantes habitan en la cabecera municipal.

${ }^{2}$ La cobertura es resultado del acceso de niños y jóvenes al sistema educativo, así como de su permanencia en él. El número de estudiantes que se escolariza es muy superior al número de aquellos que terminan un ciclo y al de quienes llegan al nivel de media. La permanencia, a su vez, no es siempre continua, lo que alarga el tiempo necesario para cumplir el ciclo7. (MEN, Manual para la Formulación y ejecución de Planes de Educación Rural, 2012, p. 7)

Volumen 1. Número 2. Julio - Diciembre 2021 


\begin{tabular}{|c|c|c|c|c|}
\hline & \multicolumn{2}{|l|}{ Cabecera } & \multicolumn{2}{|l|}{ Rural } \\
\hline & 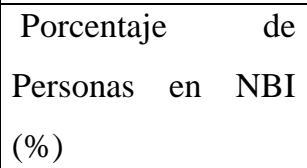 & $\begin{array}{l}\text { Porcentaje de } \\
\text { Personas en miseria }\end{array}$ & $\begin{array}{llr}\text { Porcentaje } & \text { de } \\
\text { Personas en } & \text { NBI } \\
(\%) & & \end{array}$ & $\begin{array}{l}\text { Porcentaje de } \\
\text { Personas en miseria }\end{array}$ \\
\hline ARAUCA & 27,82 & 8,15 & 55,02 & 18,82 \\
\hline
\end{tabular}

Tabla 2. Porcentaje de personas con NBI y en miseria en el municipio de Arauca Fuente: Elaboración propia a partir de datos DANE.

El estado del tema educativo en el municipio de Arauca, conforme los datos de la Secretaria de Educación Departamental (SED), extractados del Sistema de Matrícula Estudiantil de Educación Básica y Media (SIMAT) al cierre de Octubre de 2019, el departamento suma 65.870 estudiantes, y el sector oficial presenta el $94.05 \%$ de la población estudiantil, distribuidos en 67 establecimientos educativos oficiales, y 32 no oficiales, siendo el municipio de Arauca, quien presenta la mayor población estudiantil tanto en sector público como en el privado.

Los establecimientos educativos ubicados en la zona urbana del departamento representan el $63 \%$ y para la zona rural el 37\%. El municipio de Arauca presenta el 38,01\% de establecimientos ubicados en zona urbana. La cifra reportada por la Secretaría de Educación Departamental, consolida los datos reportados por cada una de las instituciones educativas, a través del SIMAT- y se evidencia una tasa de cobertura muy similar a la cobertura nacional.

\begin{tabular}{|l|l|l|l|l|}
\hline $\begin{array}{l}\text { COBERTU } \\
\text { RA_NETA }\end{array}$ & $\begin{array}{l}\text { COBERTURA_NET } \\
\text { A_TRANSICIÓN }\end{array}$ & $\begin{array}{l}\text { COBERTURA_NE } \\
\text { TA_PRIMARIA }\end{array}$ & $\begin{array}{l}\text { COBERTURA_NET } \\
\text { A_SECUNDARIA }\end{array}$ & $\begin{array}{l}\text { COBERTURA_N } \\
\text { ETA_MEDIA }\end{array}$ \\
\hline $96,70 \%$ & $54,15 \%$ & $95,80 \%$ & $74,95 \%$ & $39,84 \%$ \\
\hline
\end{tabular}

Tabla 3. Tasa de cobertura educativa en el municipio de Arauca Fuente: MEN, SIMAT, 2019

Es evidente que existe una brecha entre la cobertura del acceso a la educación de los niños y jóvenes del municipio de Arauca frente al municipio de Bogotá, evidenciando que un 19\% por encima esta la cobertura en la educación prescolar, para el nivel primaria se mantiene la 
cobertura más alta el municipio de Arauca; sin embargo, existe una tendencia de disminución de cobertura en el básica secundaria Bogotá logra mantener cobertura constante del tránsito de la primaria a la secundaria.

Para el caso del municipio de Arauca, se evidencia que decrece de manera significativamente la cobertura de la básica secundaria y en la media vocacional se evidencia una caída del $40 \%$ identificado que la cobertura neta en secundaria está muy lejos aún de ser; aceptable frente a Bogotá y pone de presente los factores socioeconómicos que hacen que haya una alta deserción escolar en el nivel de la media vocacional antes de terminar el bachillerato.

Estas cifras indican que en la medida que los niños y jóvenes crecen disminuye el acceso a la educación media vocacional, indicando que se pierde cobertura y, por ende, el número de estudiantes que termina educación media es inferior a quienes terminan básica primera en el municipio de Arauca.

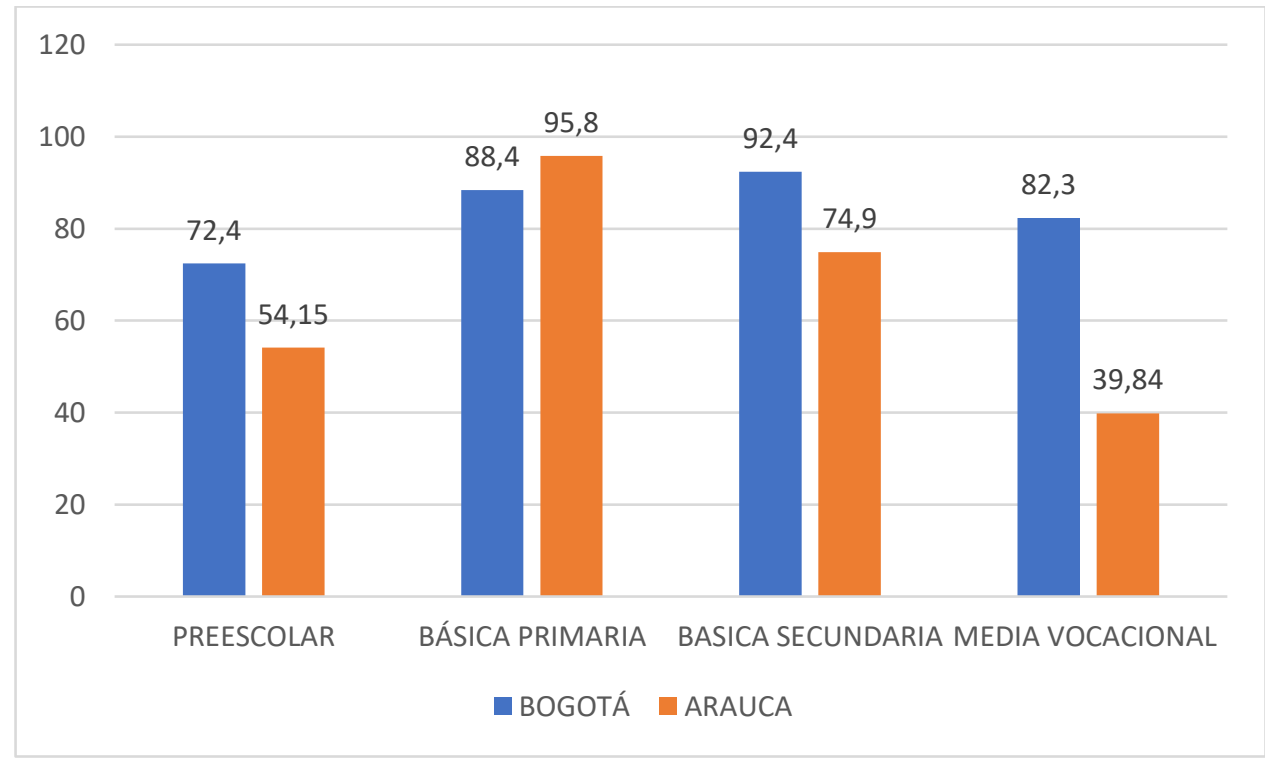

Gráfico 2. Cobertura de Educación en Bogotá y Arauca

Fuente: Elaboración propia a partir del SIMAT, 2019. Consultado en abril y mayo de 2020. 
Las cifras anteriores, evidencia que Bogotá lograr sostener una cobertura a través de todo el ciclo de educación básica primaria y secundaria, y que existe una brecha muy significativa frente al caso del municipio de Arauca.

Frente a la situación de la Pandemia mundial del COVID-19 y a la declaratoria de Pandemia de la Organización Mundial de la Salud -OMS- y siguiendo los parámetros internacionales. El presidente de Colombia el 12 de marzo de 2020 decide declarar la Emergencia Sanitaria de la mano de las directrices de la OMS para todo el territorio nacional y el Ministerio de Salud y Protección Social emite Resolución NÚMERO 385 DEL 12 DE MARZO DE 2020. "Por la cual se declara la emergencia sanitaria por causa del coronavirus COVID-19" Y a partir de dicho momento, el Ministerio de Educación Nacional emite el decreto nro. 470 de 2020 "Por el cual se dictan medidas que brindan herramientas a las entidades territoriales para garantizar la ejecución del Programa de Alimentación Escolar y la prestación del servicio público de educación preescolar, básica y media, dentro del Estado de Emergencia Económica, Social y Ecológica" y en ese momento los niños y jóvenes dejan de asistir a la jornada regular de los colegios públicos y privados de todo el país que incluyó a todos los niveles de educación en Colombia.

Dicho hito histórico para el país genera el reto más grande para todos los sectores, pero en especial, para el sector educativo, en especial, porque al cambiar del modelo presencial a la presencialidad mediada por tecnología pone en evidencia la brecha existen entre la educación pública vs. la educación privada, entre la educación de las cabeceras municipales y las zonas rural. Y, sobre todo, el acceso de los niños y jóvenes al internet y la tecnología en zonas apartadas del país, también, evidencia la desigualdad en la que viven muchos de los territorios a nivel nacional con un apoyo casi nulo del gobierno nacional.

Según los datos del Ministerio de Educación en Colombia, el número de estudiantes promedio por Computador en Colombia es de 8 estudiantes por un computador, ese dato se construyó a partir de los computadores reportados por las Secretarías de Educación Certificadas, sobre la 
matrícula total validada por el Ministerio de Educación Nacional, en la siguiente gráfica se evidencia la tendencia en los últimos nueve años.

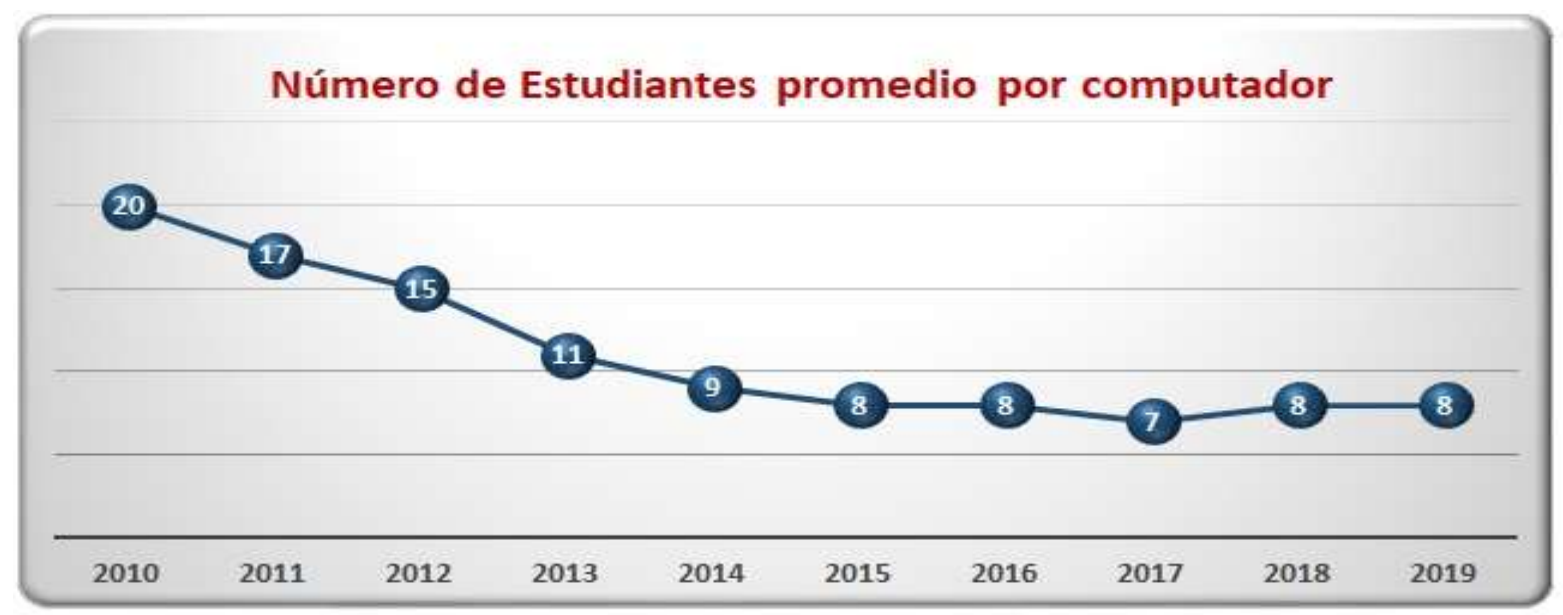

Gráfico 3. Estudiantes por computador promedio nacional

Fuente: Ministerio de Educación Nacional, consultado en mayo de 2020

Frente a la situación del aislamiento colectivo y la formación que empezaron a recibir los niños y jóvenes de los colegios públicos del sistema escolar colombiano puso en evidencia una brecha, no sólo por el acceso a los computadores sino por la conectividad de las regiones. Evidenciaría que existe una desigualdad educativa entre el centro del país y la periferia como el caso en Arauca y Bogotá. En suma, se podría pensar que, uno de los factores que influye en la desigualdad educativa es el área geográfica donde se encuentra ubicada la institución educativa, los siguientes indicadores coinciden en la información, por ejemplo, al hacer el análisis de los datos reportados por el Ministerio de las TIC y el MEN, se encuentra que:

En la ciudad de Bogotá, según el programa conexión total del Ministerio de Educación existen 731 sedes educativas de las cuales se encontraban conectadas en a marzo de 2020 el 93,17\% y que a mayo se logró la conectividad del 100\%, ver gráfico siguiente: 

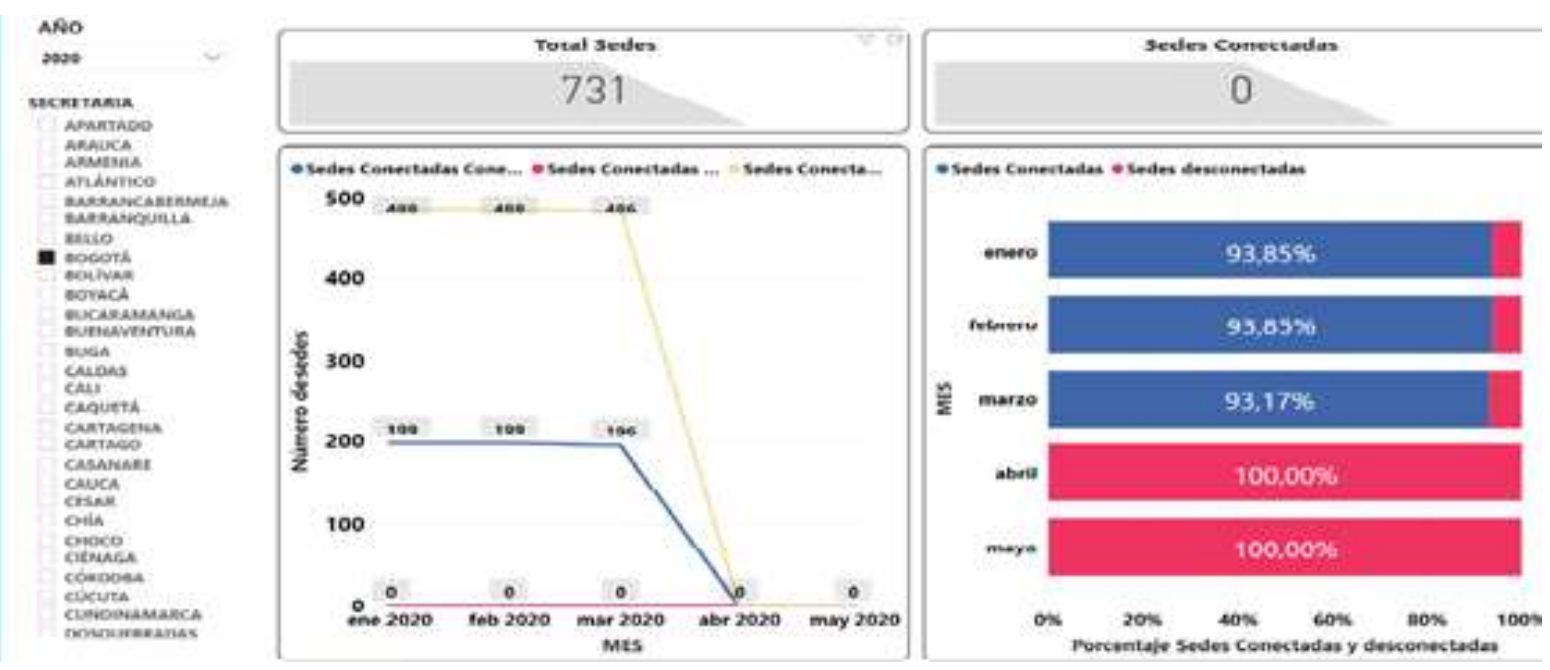

Gráfico 4. Número de instituciones educativas conectadas a mayo de 2020

Fuente: MEN. Programa conexión total, mayo de 2020.

Para el caso del municipio de Arauca, se evidencia que de las 471 que solo habían conectadas el 15\%, una diferencia enorme frente a la conectividad que existe en la ciudad de Bogotá, lo que pone de presente que existe una muy baja conectividad en el municipio de Arauca, y, por ende, no existen condiciones de igual para recibir la educación a través de las plataformas tecnológicas. 


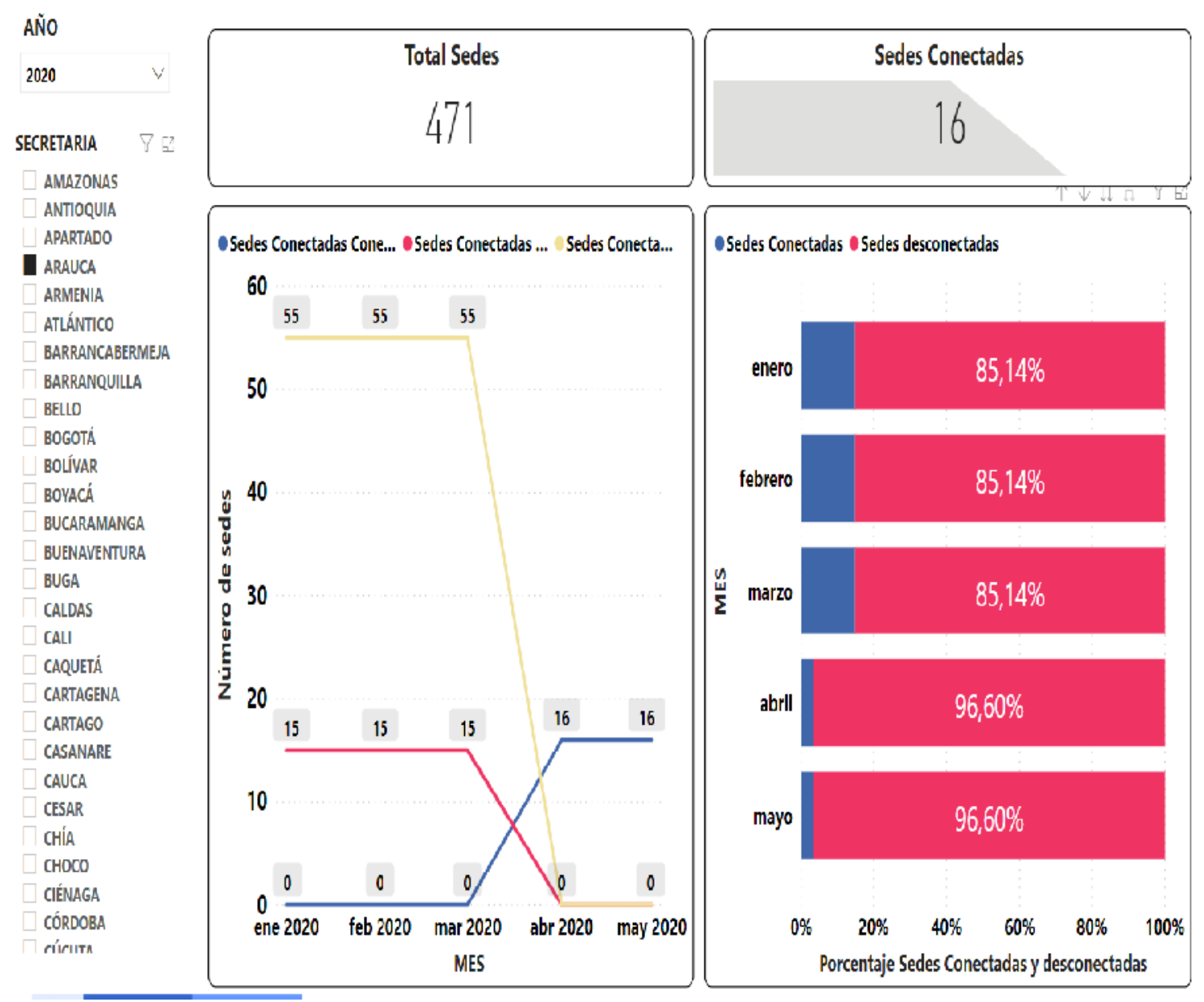

Gráfico 5. Número de instituciones educativas conectadas a mayo de 2020, para el municipio de Arauca Fuente: MEN. Programa conexión total, mayo de 2020.

Otro de los programas liderado por el Gobierno Nacional denominado "Computadores por Educar", cuyo objetivo es el programa del Gobierno Nacional de mayor impacto social que genera equidad a través de las TIC, fomentando la calidad de la educación bajo un modelo sostenibilidad; evidencia que el municipio de Arauca, hay en promedio un computador por diez (10) estudiantes, con corte de cifra a diciembre de 2019 y para el caso de Bogotá, la cifra es mucho menor un (1) computador por tres (3) estudiantes. El promedio de computadores de escritorio por hogar para el total nacional fue de 0,22, según la encuesta de hogares del DANE. Por su parte, el promedio de computadores portátiles y tabletas por hogar fueron de 0,36 y 0,13 respectivamente (ver Gráfico siguiente nro.6). 


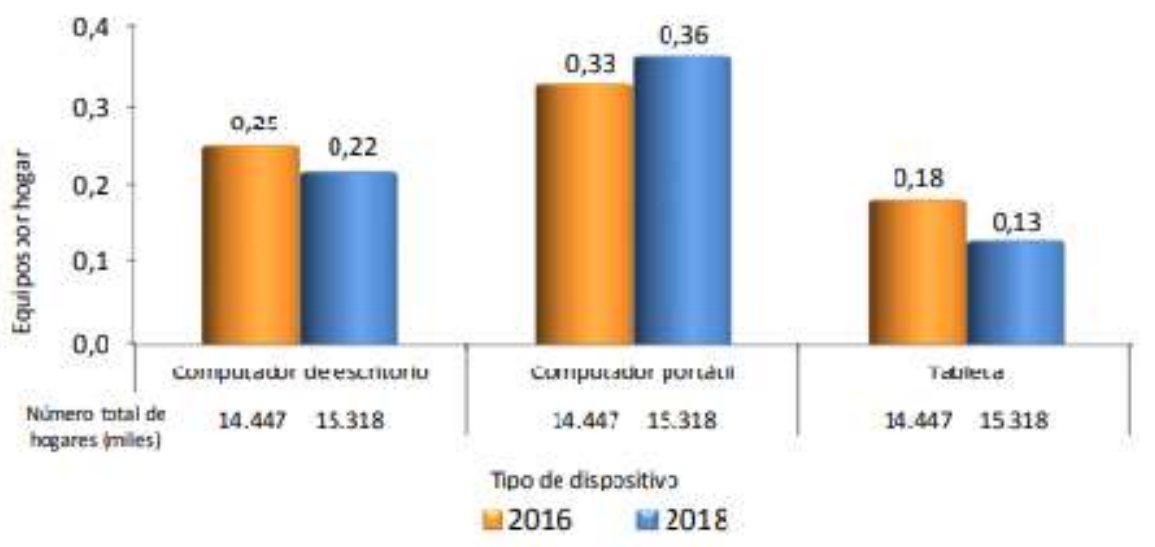

Gráfico 6. Tenencia y uso de tecnologías de la información y comunicación - TIC en hogares Fuente: DANE, 2018. Indicadores básicos de tenencia y uso de tecnologías de la información y comunicación - TIC en hogares y personas de 5 y más años de edad.

Los anteriores datos, evidencia la necesidad de problematizar el asunto de la alternancia en las instituciones educativas y de particular las políticas, lineamientos y protocolos emitidos por el Ministerio de Educación Nacional en el marco de la pandemia del Covid-19, no sólo por la evidencia estadística sino por las condiciones como se hacen los lineamientos y protocolos, podría asegurar si se hace un levantamiento de información primeria se podría revelar que son muchos los factores y condiciones para demostrar que existen causas estructurales de la mirada sobre la educación y los artefactos que se requieren para explicar la desigualdad educativa y territorial que se reproduce en los lineamientos muy soterrada en una idea de homogeneidad con ideas que la desigualdad es un asunto residual y no central en los diseños pedagógicos, didácticos como en el caso de nuevas formas de garantizar el derecho a la educación, por tanto, debe interpelar esa idea de unificación e homogeneización de la política tanto para los niños de las zonas urbanas como rurales, tanto para los del centro que para los de la periferia, y también cuestionar esa noción de igualdad. Sin perder de vista la importancia de comprender que la igualdad es fundamental y ausente, es actual e intempestiva, siempre atribuida a la iniciativa de los individuos y de grupos que, contra el curso ordinario 
de las cosas, asumen el riesgo de verificarla, de inventar las formas, individuales o colectivas, de su verificación y hoy más que nunca se requiere dicha mirada.

Por tanto, es fundamental reconocer la idea de igualdad y justicia que se presenta en el modelo de alternancia, como la opción emitida por la Ministra de Educación María Victoria Angulo, que da respuesta a la reactivación del sistema educativo colombiano, por tanto, es necesario revisar las nociones de escuela y reconocer la ponderación multidimensional y multifactorial de las dinámicas propias en cada institución educativa y teniendo en cuenta los patrones sociales y culturales de cada territorio.

\section{El modelo de alternancia para reactivar al sector educativo}

Para emancipar al otro, es necesario estar emancipado uno mismo. "Hay que conocerse a sí mismo como viajero del espíritu, al igual a todos los demás viajeros, en cuanto a sujeto intelectual que participa del a potencia común de los seres intelectuales" (Ranciére, 2018, p. 66).

En el marco de la situación actual -aislamiento colectivo obligatorio en el territorio colombiano-, y a lo largo de los meses de aislamiento en casa, el ente rector de la educación ha emitido una serie de lineamientos, entre otros, la Circular Conjunta número 11 con el Ministerio de Salud y Protección Social del 9 de marzo de 2020 con recomendaciones para prevención, manejo y control de la infección respiratoria aguda por el nuevo coronavirus en el entorno educativo; las Circulares 19, 20 y 21 del 14, 16 y 17 de marzo de 2020 y las Directivas 3, 5, 7, 9, 10, 11 y 12 del 20 y 25 de marzo, del 6 y 7 de abril, del 29 de mayo, con el fin de orientar las actividades del sector en medio de la disposición de aislamiento preventivo obligatorio, las cuales se han encaminado a generar las condiciones para que niñas, niños y adolescentes continúen su proceso educativo en casa con la guía y acompañamiento pedagógico de sus maestros y la mediación de sus familias.

Volumen 1. Número 2. Julio - Diciembre 2021 
Y para continuar en la protección de la vida y la integridad de la vida de los niños y jóvenes, el Ministerio de Educación Nacional expidió la Directiva 012 con fecha del 02 de junio de 2020; que contiene las orientaciones para las autoridades competentes en educación de los departamentos, distritos y municipios, así como para los rectores de los establecimientos educativos no oficiales (jardines infantiles y colegios privados), dirigidas a la continuidad en la prestación del servicio público de educación inicial, preescolar, básica y media. Dicha directiva señala que según la evolución epidemiológica de la pandemia por el COVID-19 y disposiciones de las autoridades durante la emergencia sanitaria, se amplía la prestación del servicio educativo en casa para la población estudiantil de los niveles de educación inicial, preescolar, básica (primaria y secundaria) y, media de establecimientos educativos no oficiales en el modelo alternancia.

Sin embargo, el modelo no es claro para las diferentes regiones, entendiendo que el último censo del Dane (2018), solo el 43 por ciento de las personas tienen acceso a internet móvil o fijo y que el modelo está muy planteado en los artefactos y los dispositivos tecnológicos.

En este sentido, considero que, a fin de erradicar esas situaciones de desigualdad, es necesario precisar las obligaciones que tienen los Estados a la hora de hacer efectivo este derecho desde una mirada igualitaria- igualdad real de oportunidades (Rawls, 2016). El mandato de igualdad de oportunidades implica interpretar la garantía de acceso como algo más que la igualdad formal. (Rodino, 2011) y como lo señala Martinis, y han dejado de lado la visión del docente como lo plantea Southwell (2016):

desarrollan definiciones vinculadas a qué situaciones de las que viven cotidianamente son del orden de la desigualdad, la injusticia y la exclusión, y cómo la escuela y el trabajo docente podrían tener un lugar en su posible resolución (...), también, el vínculo afectivo con las comunidades académicas y la circulación de discursos sobre la tarea de enseñar, en el marco de lo cual se vuelve central el análisis de los sentidos que las acciones, disposiciones e instituciones tienen para quienes las viven. (p. 168) 
Dicho de otro modo, el modelo de alternancia no incluyó a los docentes como sujetos vivos e históricos que están en el centro de la implementación y totalmente invisible en el discurso desposeídos de todo y olvidando que es el docente. Southwell (2014) "los múltiples modos en que los sujetos enseñantes asumen, viven y piensan su tarea, y los problemas, desafíos y utópicas que se plantean en torno de ella" (p. 166), por tanto, la importante de ser reconocidos y con un papel central en el modelo de alternancia.

Dicho concepto de alternancia:

está referido a la prestación del servicio educativo mediante la conjugación de distintas variables y circunstancias presentes en la operación del mismo, de acuerdo con las posibilidades de la población, de la institución y del territorio. La combinación de estrategias puede contemplar diferentes opciones: alternancia del trabajo educativo que han apoyado las familias en casa con el acompañamiento de los maestros, complementado con encuentros periódicos presenciales e integración de diversos recursos pedagógicos; alternancia de la asistencia a la institución educativa por parte de grupos específicamente organizados o priorizados; alternancia del uso de espacios en la institución educativa o de la comunidad; alternancia entre la medida de retorno progresivo y la medida de permanecer exclusivamente en el trabajo académico. (MEN, 2020, p. 20)

Lo anterior, pone en tensión al sistema educativo donde existen brechas de toda índole y, por tanto, este artículo evidencia que existe una desigualdad educativa, entendiendo la definición planteada por Subirats, Gomá y Brugué (2005), la desigualdad educativa consiste en excluir a determinados individuos o colectivos del acceso, permanencia y aprovechamiento del sistema educativo; pero no sólo pensarlo en las herramientas tecnológicas sino un asunto más allá del tema cognitivo, tal vez, a travesado por el tema ético. También, no sólo el pensar en el terreno de lo geográfico sino de la condición socioeconómica de los niños y jóvenes y el modelo propuesto está basado en una política que establece que todas instituciones educativas tienen el mismo punto de partida, se podría decir, que está centrado en la igualdad de 
oportunidades, una mirada muy instaurada en la sociedad colombiana el logro a través del mérito:

Consiste en ofrecer a todos la posibilidad de ocupar las mejores posiciones en función de un principio meritocrático. Quiere menos reducir la inequidad entre las diferentes posiciones sociales que luchar contra las discriminaciones que perturbarían una competencia al término de la cual los individuos, iguales en el punto de partida, ocuparían posiciones jerarquizadas. (Dubet, 201, pág. 12)

Es evidente que los jóvenes habitantes de los territorios como Arauca, con una distancia geográficamente de 720 kilómetros distante del centro del país, con un índice de NBI muy bajo, y con cifras de cobertura muy por debajo de la media nacional no están en el mismo punto de partida y en consecuencia muchos abandonaran el sistema educativa y no se habrá garantizado el derecho a la educación, no sólo por la falta de elementos tecnológicos sino por las incomprensiones de las dinámicas que requieren cambios y ajustes de la estructura, y tampoco, tienen los mismos accesos ponen de presente que el modelo de alternancia propuesto por el MEN es un modelo desigual e injusto que genera más inequidad de la existente.

Al revisar en detalle el lineamiento planteado por el MEN, pone su acento en una igualdad para todos en la medida que establece unos criterios de bioseguridad homogéneos para todos, y cualquier distraído se interroga que pasa con las comunidades étnicas, los niños de las escuelas rurales con horas de camino y con dificultades para los desplazamientos, los requisitos o condiciones que a continuación enumerar son medidas universales para diferentes servicios para la sociedad, entre ellos los siguientes:

- Asistencia al servicio educativo siempre y cuando no se presente ningún síntoma de enfermedad. 
. Uso de tapabocas que cubra nariz y boca para su uso correcto revisar los Lineamientos generales para el uso de tapabocas convencional y máscaras de alta eficiencia del Ministerio de Salud y Protección Social ${ }^{3}$.

- Los tapabocas pueden ser fabricados por las familias y/o cuidadores según las recomendaciones dadas en la guía del Ministerio de Salud y Protección Social ${ }^{4}$.

. Establecer un horario específico de lavado de manos con agua y jabón mínimo cada 3 horas preferiblemente bajo supervisión de un adulto. (antes y después de consumir alimentos, cuando se vean visiblemente sucias, antes de ingresar a la clase después de los recesos).

- Higienización con gel antibacterial o alcohol glicerinado si hay contacto con alguna superficie diferente a la de su mobiliario personal o hubo desplazamiento a otra aula.

. Abstenerse de compartir alimentos y materiales como expresión de cuidado de sí mismo y del otro.

. Evitar tocarse la cara, frotarse la nariz y los ojos.

. Mantener distancia de 1 a 2 metros entre las personas en la interacción con ellas. ${ }^{5}$

. Es indispensable que en cada espacio o área de la institución educativa se garantice este distanciamiento físico. De acuerdo a esta consideración definir el número de personas por espacio.

. Durante el consumo de alimentos:

Realizar lavado de manos de mínimo 20 segundos con agua y jabón

En ellos se hace evidente, que las políticas públicas emanadas desde arriba top down pierden de foco la desigualdad territorial, social y estructural; en la que se encuentran muchos docentes y niños y jóvenes del territorio nacional, en especial, del municipio de Arauca y que se vuelven reproductoras de la visión del mundo dominante y por tanto, muchos niños

\footnotetext{
${ }^{3}$ Seguir las recomendaciones dadas en el Lineamiento general para el uso de tapabocas convencional y mascarillas de alta eficiencia disponible en: https://www.minsalud.gov.co/Ministerio/Institucional/Procesos\%20y\%20procedimientos/GIPS18.pdf

${ }^{4}$ Guía con lineamientos mínimos para la fabricación de tapabocas de uso general no hospitalario en el marco de la emergencia sanitaria por enfermedad COVID-19 disponible en: https://www.minsalud.gov.co/Ministerio/Institucional/Procesos\%20y\%20procedimientos/GMTG15.pdf ${ }^{5}$ Consideraciones para las medidas de salud pública relativas a las escuelas en el contexto de la COVID-19.OMS. https://apps.who.int/iris/bitstream/handle/10665/332107/WHO-2019-nCoV-Adjusting_PH_measures-Schools2020.1-spa.pdf
}

Volumen 1. Número 2. Julio - Diciembre 2021 
terminarán excluidos del sistema formal de educación en la medida que no cuentas con los artefactos para atender las clases mediadas o acompañadas por las tecnologías y continua fallando las políticas de inclusión social porque se quedaron con una idea falsa y vieja que los docentes no cuentan con la formación para atender estos desafíos. Adicional, según los mismos datos del MEN, en las zonas apartadas del país, Esta situación ilustra que las Instituciones Educativas ubicados en zonas con menos recursos económicos (que suelen estar en zonas rurales o en comunidades indígenas).

Por ende, se puede afirmar que los lineamientos del MEN, son injustos, no sólo porque privilegian a los niños y jóvenes con mayor acceso a conectividad y uso de tecnología, así como docentes con mayor nivel de apropiación de los recursos tecnológicos, y por tanto, se seguirá manteniendo en la sociedad una diferenciación de clase que privilegia a las clases socioeconómicas más altas y con contenido pedagógico más actualizado, con una mirada desde los escritorios de los hacedores de política pública que cuentan con otras trayectorias; podrían darse muchos ejemplos en el marco de la reactivación por ejemplo: El nro. de estudiantes por metro cuadrado en las instituciones públicas, hoy antes de la pandemia asisten entre 40 y 45 niños a un salón que mide $6 \mathrm{~m}$ de largo, 5 metros de ancho y 3 metros de altura y para lograr el modelo de alternancia es necesario mantener una distancia de entre 1 y 2 metros lo que indica que sólo un cuarto de estudiantes podrán asistir por curso, adicional, muchos de los niños por la condición socioeconómica de los padres no cuentan con los recursos para comprar los insumos adicionales como tapabocas, alcohol, entre otros, la pregunta central sería, ¿Cuáles son los ajustes institucionales y los lineamientos para el cuidado de la vida de los docentes, niños y niñas?, el mismo modelo desarrollo no debe plantear la necesidad de buscar sociedades libres.

\section{Conclusiones}

Los casos observados frente a la desigualdad educativa geográfica -Bogotá y Araucaevidencia brechas existen y estructurales necesarios de ahondar y comprender, sin embargo, más allá de dichas cifras se hace necesario traspasar el análisis del artefacto tecnológico por

Volumen 1. Número 2. Julio - Diciembre 2021 
el tema de hacer análisis de la igualdad social desde lo colectivo y como se comprende el derecho de la educación. En primer lugar, es necesario para lograr la reactivación del sector educativo es fundamental, reavivar la escuela y los sujetos que la habitan; en términos de (Dubet, 2011) un contrato de solidaridad ampliada, tal como lo propone desde actos y principios modestos, efectivos y realistas. Para producir la solidaridad, es necesario ampliar la democracia, refundar las instituciones y lograr que los individuos se reconozcan como solidarios, aún antes de aceptarse como iguales y diferentes. Y que la práctica para volver a la escuela parta de la pregunta ¿Qué piensas?, todo su poder se encuentra en la conciencia de emancipación que actualiza en el maestro y suscita en el alumno (Ranciére, 2018), podría pensarse como soñador, pero considero que es el momento de retejer las historias y así le apostamos a temas más allá de procedimientos vacíos de significado.

De otra parte, es ineludible, entender que hay diferencias en cada territorio y múltiples formas de afrontar los desafíos y que no existe una única forma para realizarlo y a su vez, de descentrar discursos instaurados con la modernidad que hacen mucha presencia en las prácticas cotidianas de las escuelas y en los lineamientos de política pública y evidencian lo instrumental que está el sistema educativo colombiano, de ahí la importante de pensar en los sujetos que habitan la escuela, no sólo por el significado sino como se logra salir de las practicas cotidianas y estar dispuesto a ser emancipadores en sus entornos, desde el docente como las lecturas de las instituciones educativa y lo afectivo como proceso para descentrar prácticas procedimentales.

Y tal como lo planteó Ranciére (2018), producto de la pandemia la desigualdad no puede pensarse a sí misma, de allí el desafío de construir puentes para establecer reconocimiento de la diferencia e identificar esa noción de educabilidad planeada por Martinis (2016) y que a partir de la vivencia se posibiliten la construcción de sentidos en términos pedagógicos en los entornos educativos y no desde las carencias, sino la posibilidad de abrir espacio de discusión pública con docente y estudiantes para que el discurso de la alternancia pase de lo procedimental a comprensiones más amplias sobre los factores que mantienen y denotan las desigualdades educativas. 


\section{Referencias}

Chiroleu, A. (2012). Políticas de Educación Superior en América Latina en el Siglo XXI: ¿Inclusión o Calidad? Education Policy Analysis Archives/Archivos Analíticos de Políticas Educativas, 20, 1-16

Chiroleu, A. (2013). Políticas públicas de Educación Superior en América Latina: ¿democratización o expansión de las oportunidades en el nivel superior? Espacio Abierto, 22 (2), 279-304.

Dubet, F. (2011). Repensar la justicia social: contra el mito de la igualdad de oportunidades. Argentina: Siglo XXI.

Dubet (2012). Los límites de la igualdad de oportunidades. Nueva sociedad 239, p. 42-50. Argentina.

DANE. (2018) Boletín Técnico Indicadores básicos de tenencia y uso de tecnologías de la información y comunicación - TIC en hogares y personas de 5 y más años de edad. Consultado en: https://www.dane.gov.co/files/investigaciones/boletines/tic/bol_tic_hogares_2018.pdf Departamento Administrativo Nacional de Estadísticas -DANE-. «Cuentas departamentales». Consultado el 26 de mayo de 2020. https://www.dane.gov.co/index.php/estadisticas-portema/cuentas-nacionales/cuentas-nacionales-departamentales

DANE, https://www.dane.gov.co/index.php/estadisticas-por-tema/demografia-ypoblacion/ proyecciones-de-poblacion consultado en mayo de 2020.

Martinis, P. (2016). Aproximación a los usos del significante "inclusión educativa" en la formulación de políticas educativas en Uruguay (2005-2015). Diálogos entre Argentina, Brasil y Uruguay. Sujetos, políticas y organizaciones en educación (pp. 1-15). Buenos Aires, Biblos. 
MEN. (2020). Programa conexión total. Consultado en mayo de 2020: https://www.mineducacion.gov.co/portal/micrositios-institucionales/ConexionTotal/Conexion-Total-en-Cifras/348154:Porcentaje-de-Matricula-oficial-con-conexion-ainternet

Molina G. H. (1996) Ciudad y Región en Colombia. Bogotá, la ocupación a largo plazo. Universidad Externado de Colombia. pp. 302

Parsons, W. (2007). Políticas Públicas: una introducción a la teoría y la práctica del análisis de políticas públicas. Buenos Aires: FLACSO.

Passeron, Pierre y Bourdieu, Jean-Claude 1996 La reproducción: Elementos para una teoría del sistema de enseñanza (Barcelona: Editorial Laia, S.A.).

Puiggrós, A (1996): Presencias y ausencias en la historia de la historiografía pedagógica latinoamericana. En Cuccuzza, R. (coord.). Historia de la educación a debate. Buenos Aires, Miño y Dávila Editores. pp. 91-123

Subirats, J., Gomá, R., \& Brugué, J. (2005). Análisis de los factores de exclusión social. Bilbao: Fundación BBVA. Consultado en:

Southwell, M., \& Vassiliades, A. (2014). El concepto de posición docente: notas conceptuales y metodológicas. Educación, lenguaje y Sociedad, 11(11).

Rawls, J (1995), Teoría de la justicia, México, Fondo de Cultura Económica. 